\title{
Use of Linear Ion Traps in Data-Independent Acquisition Methods Benefits Low-Input Proteomics
}

\author{
Eva Borràs, Olga Pastor, and Eduard Sabidó*
}

Cite This: Anal. Chem. 2021, 93, 11649-11653

Read Online

ABSTRACT: The need for a better understanding of cellular heterogeneity has pushed mass spectrometry technologies to the analysis of single-cell and singlecell-type proteomes, although several challenges still limit their widespread implementation. Among the efforts toward single-cell and low-input analyses, there is the adoption of data-independent acquisition methods to increase analytical sensitivity. Here, we revisited the use of linear ion traps mass analyzers in data-independent acquisition methods and demonstrate their benefits to boost peptide and protein identifications in low-input proteomes.

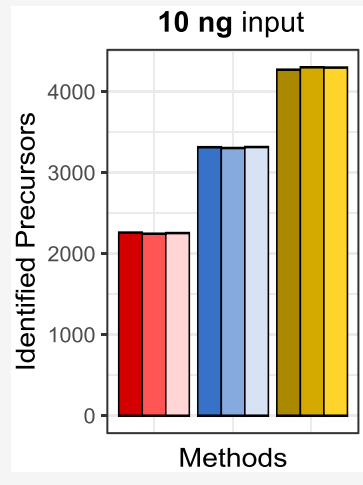

DIA-OT 40X10 30K

DIA-IT 40X10 normal

$\square$ DIA-IT 100X4 normal

ingle-cell and low-input applications have recently $\checkmark$ exploded as the means to better understand the cellular heterogeneity that drives the development of normal and pathological tissues. ${ }^{1}$ Although a plethora of methods have flourished in genomics, ${ }^{2}$ single-cell and low-input technologies still face several challenges in mass spectrometry-based proteomics, both at sample handling and data acquisition. A few efforts have successfully been made in both directions ${ }^{3-7}$ that have paved the road for these proteomics technologies, but there are still several challenges that limit their widespread implementation. ${ }^{8}$

Among the efforts toward the analysis of single-cell and single-cell-type proteomes, there is the adoption of dataindependent acquisition (DIA) methods-often combined with ion mobility separation - to increase the sensitivity during the analysis of small amounts of complex samples. ${ }^{3,6}$ DIA methods use several broadband isolation windows to co-isolate and fragment peptides within samples regardless of their signal or amount. Consequently, these methods provide a systematic sampling of fragment ions that are later used for peptide identification and quantification with high sensitivity and improved dynamic range. ${ }^{9-11}$ Despite several DIA methods were developed using linear ion traps in their origins, ${ }^{12,13}$ most DIA methods currently rely on high accuracy/high resolution mass analyzers such as time-of-flight and Orbitrap, ${ }^{14-18}$ with few exceptions. ${ }^{19}$ Linear ion traps offer fast scanning rates and high sensitivity that exceed those of high accuracy/high resolution orbital ion traps. Therefore, here we revisited the use of linear ion traps mass analyzers in DIA methods to evaluate their potential to boost peptide and protein identifications in low-input proteomics applications.
Initially, we made serial dilutions of commercially available HeLa protein digest and loaded 1, 10, 100, and $1000 \mathrm{ng}$ in a 50 $\mathrm{cm}$ nano-LC column for liquid chromatography separation and subsequent mass spectrometric analysis with an EASY-nLC 1200 coupled to an Orbitrap Fusion Lumos (Supporting Information). A gradient of $120 \mathrm{~min}$ was used to resolve the proteomes and obtain peak widths compatible with the cycle times of the evaluated methods (Table 1). All dataindependent acquisition methods tested in this study had their precursor peptides ions analyzed in a high accuracy/high resolution Orbitrap mass analyzer, whereas fragment ions generated by HCD fragmentation (NCE 28) were analyzed either in an Orbitrap or a linear ion trap mass analyzer. Data acquisition parameters were fitted to each method according to previous works (Supporting Information, Supplementary Table 1, Supplementary Table 2, Supplementary Table 3, and Supplementary Table 4). ${ }^{20-22}$

An Orbitrap-based DIA method with 40 windows of $10 \mathrm{Da}$ covering the range of $500-900 \mathrm{~m} / z$ at 30,000 resolution (DIAOT $40 \mathrm{X} 1030 \mathrm{~K}$ ) was used as the reference method and compared to equivalent methods using the linear ion trap for the analysis of the peptide fragment ions (DIA-IT 40X10). Several linear ion trap-based DIA methods (DIA-IT) at different scanning speeds were tested, i.e., normal, rapid, and

Received: May 4, 2021

Accepted: July 20, 2021

Published: August 18, 2021 
Table 1. Main Parameters for Different DIA Methods Evaluated

\begin{tabular}{|c|c|c|c|c|c|c|}
\hline & Analyzer & Scanning speed $(\mathrm{Da} / \mathrm{s})^{*}$ & MS2 Injection time (ms) & Overhead (ms) & Resolution & Cycle time (s) \\
\hline DIA-IT $40 X 10$ turbo & Ion trap & 125,000 & 12 & 10 & - & 1.1 \\
\hline DIA-IT $40 X 10$ rapid & Ion trap & 66,000 & 18.5 & 10 & - & 1.3 \\
\hline DIA-IT 40X10 normal & Ion trap & 33,000 & 32 & 10 & - & 1.9 \\
\hline DIA-IT 100X4 turbo & Ion trap & 125,000 & 12 & 10 & - & 2.5 \\
\hline DIA-IT 100X4 rapid & Ion trap & 66,000 & 18.5 & 10 & - & 3.2 \\
\hline \multirow[t]{2}{*}{ DIA-IT 100X4 normal } & Ion trap & 33,000 & 32 & 10 & - & 4.5 \\
\hline & & Measurement time (ms) & MS2 Injection time (ms) & Overhead (ms) & Resolution & Cycle time (s) \\
\hline DIA-OT $40 X 1030 \mathrm{~K}$ & Orbitrap & 64 & 54 & 10 & 30,000 & 3.5 \\
\hline DIA-OT $40 \times 1015 \mathrm{~K}$ & Orbitrap & 32 & 22 & 10 & 15,000 & 2.2 \\
\hline DIA-OT $40 \mathrm{X} 107.5 \mathrm{~K}$ & Orbitrap & 16 & 6 & 10 & 7500 & 1.6 \\
\hline
\end{tabular}

turbo (Table 1). In all methods the injection times were adjusted to match the scanning speeds and thus achieve optimal ion management. Acquired mass spectrometric data were analyzed with Spectronaut v14.7 (Biognosys) using the directDIA approach and the Pulsar search engine, ${ }^{22}$ with peptides and proteins filtered at 1\% FDR. Our results showed that linear ion trap DIA methods outcompeted Orbitrap-based DIA (DIA-OT) methods on the number of precursors identified when analyzing low amounts of sample $(<10 \mathrm{ng})$ (Figure 1A). Moreover, within the DIA-IT methods, we observed that those using slightly slower scanning rates and larger MS2 injection times resulted in a higher number of peptide identifications. These observations were further corroborated with additional triplicate analyses with $1 \mathrm{ng}$ of HeLa tryptic digest (Figure 1B). These analyses confirmed that the higher sensitivity exhibited by linear ion traps in DIA methods are beneficial in terms of peptide identification in low-input proteomes. Beyond the increase in the number of peptide identifications, we further evaluated whether DIA-IT methods recapitulated the same results than DIA-OT methods in terms of identified peptides and their quantification. Our results showed an excellent overlap between DIA-IT and DIAOT methods in the set of identified peptide sequences (Figure 1C). To evaluate the correctness of the peptide identifications, we corroborated that the precursors identified in low-input samples with the DIA-IT method correspond to the most abundant peptides within the sample (Supplementary Figure $1 \mathrm{~A}$ ), and we confirmed that their retention time values highly correlate among the different assessed methods (Supplementary Figure 1B). Moreover, we inspected the pseudo-MS2 spectra created from the directDIA algorithm. Not only are the spectra obtained in low-input samples with DIA-IT methods comparable to those obtained with DIA-OT (Supplementary Figure 2), but the ion-trap spectra have in general more annotated fragment ions than those spectra resulting from Orbitrap-based DIA methods when analyzing low-input samples (Supplementary Figure 3). In terms of peptide quantification, our results showed similar or even better distribution of coefficient of variation in DIA-IT triplicate analyses (Figure 1D) and a very good correlation in terms of peptide quantification (Figure 1E). Finally, it is worth noting that even the slowest evaluated DIA-IT method (DIA-IT $40 \mathrm{X} 10$ normal) exhibited reduced cycle times (1.1 s in turbo, $1.3 \mathrm{~s}$ in rapid, and $1.9 \mathrm{~s}$ in normal) compared to the reference DIA-OT method (3.5 s) and increased number of average sampling points per chromatographic peak (Figure 1F, Supplementary Figure 4A).

We reasoned that if the improvement in peptide identification observed in low-input proteomes was mainly due to higher sampling rates, a similar effect could be achieved by reducing the mass resolution in the DIA-OT methods and thus obtain enough data points in low-intensity chromatographic peaks. We therefore tested variants of the DIA-OT method with reduced resolution $(15 \mathrm{~K}$ and $7.5 \mathrm{~K})$ and adjusted injection times for optimal ion management. These Orbitrapbased DIA variants exhibited shorter cycle times $(2.2 \mathrm{~s}$ in $15 \mathrm{~K}$, $1.3 \mathrm{~s}$ in $7.5 \mathrm{~K}$ ) and higher sampling rates than the reference DIA-OT method. However, the use of low-resolution, fastscanning DIA-OT methods proved to be less sensitive in all the amounts of the measured HeLa tryptic digest (1-1000 ng) (Supplementary Figure 4B). Indeed, these methods performed worse in terms of peptide identifications than the linear ion trap-based methods, and any gain achieved from faster scanning speeds could not compensate the combination of low resolution, smaller companion injection times and the lower sensitivity of the Orbitrap mass analyzer.

Next, we wanted to confirm that the observed benefits of linear ion trap-based DIA methods were independent of the isolation scheme used. For this purpose, we adapted the recently published DIA + method $^{23}$ by setting the linear ion trap as the mass analyzer for peptide fragments and matched the MS2 injection times for optimal method synchronization (Supporting Information). DIA+ is a data-independent acquisition method with nonsequential multiplexed isolation windows that combine signals from identical peptides with different charge states, and therefore, it differs considerably from the isolation scheme previously evaluated. We performed triplicate analyses of $1 \mathrm{ng}$ of HeLa tryptic digest with the reference Orbitrap-based DIA+ method and compared it to the equivalent methods using the linear ion trap for the analysis of the peptide fragment ions. Our results showed that the linear ion trap-based DIA+ methods also outperformed the reference Orbitrap-based DIA+ method in terms of peptide identifications. Despite the intrinsic lose of specificity of larger windows, the benefits of the DIA-IT method are still superior to the potential limitations of low-resolution analyzers to resolve complex spectra. These results, together with recent reports on the use of ion trap-based parallel reaction monitoring using narrow windows, ${ }^{24}$ confirm that the observed benefits of the linear ion trap do not strictly depend on the isolation scheme used (Supplementary Figure 4C).

Finally, given the low cycle time exhibited by the evaluated DIA-IT methods, we assessed whether we could dedicate some additional time to further improve the specificity of the DIA-IT methods without compromising their cycle time. Indeed, the inherent low resolution of linear ion traps limits their specificity, which is one of their main disadvantages when compared to high-resolution mass analyzers. For this purpose, 


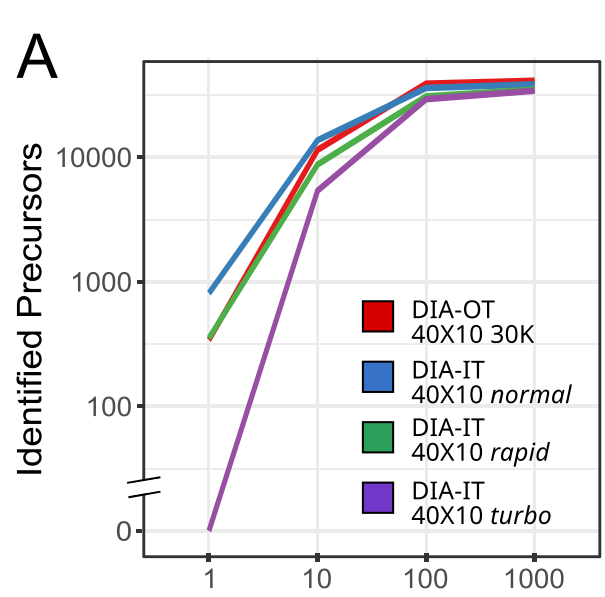

Amount (ng)

D
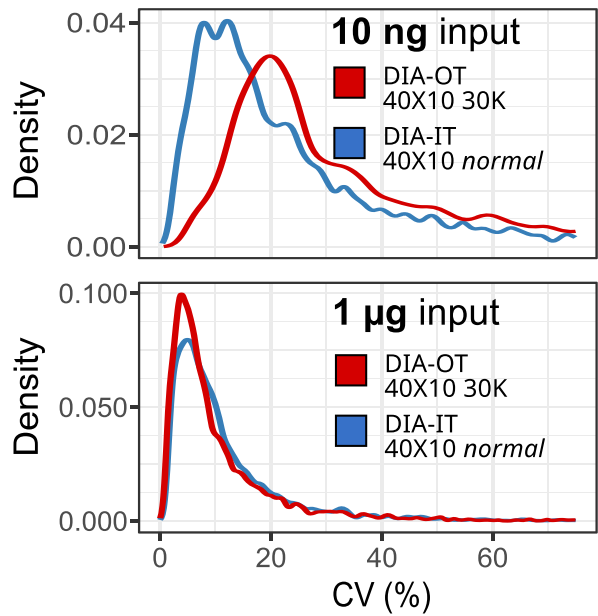

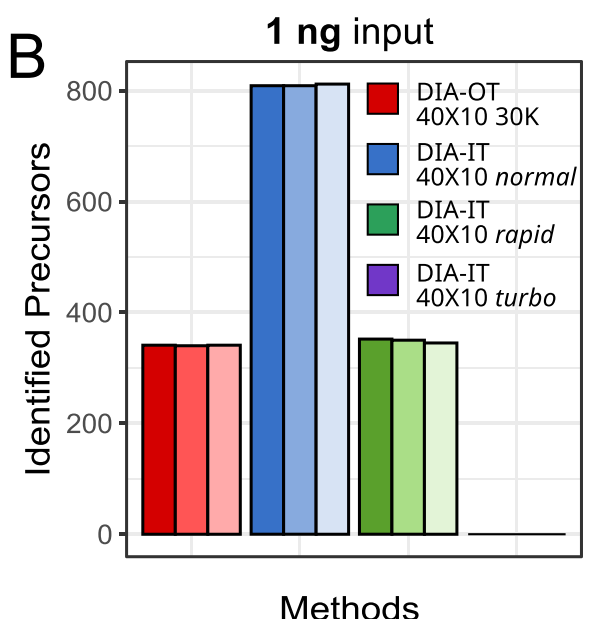

Methods

E

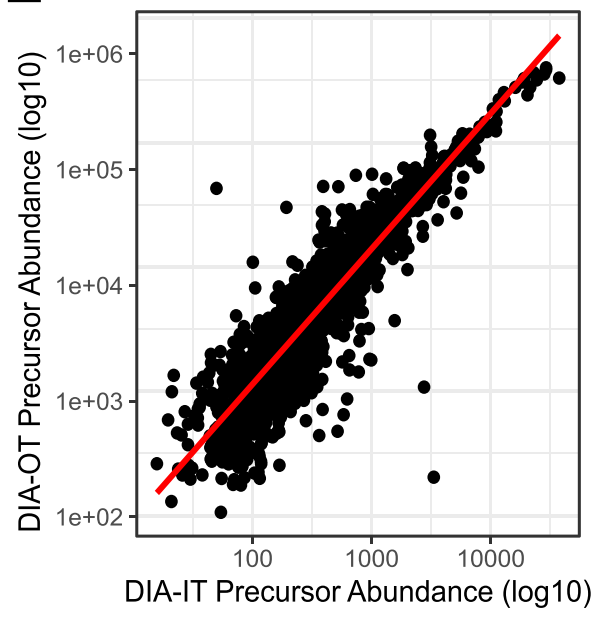

C

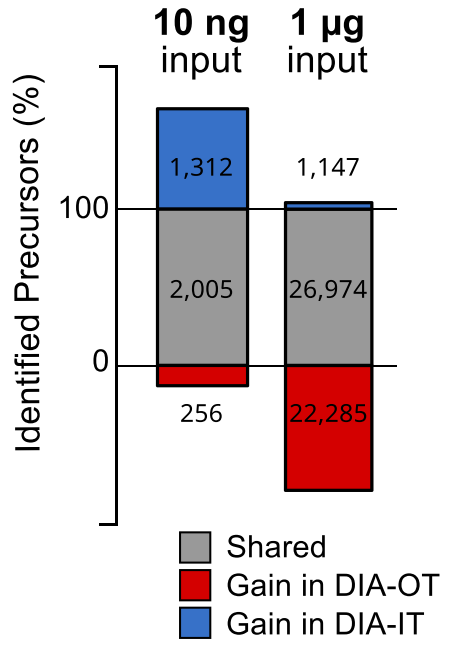

$\mathrm{F}$

10 ng input

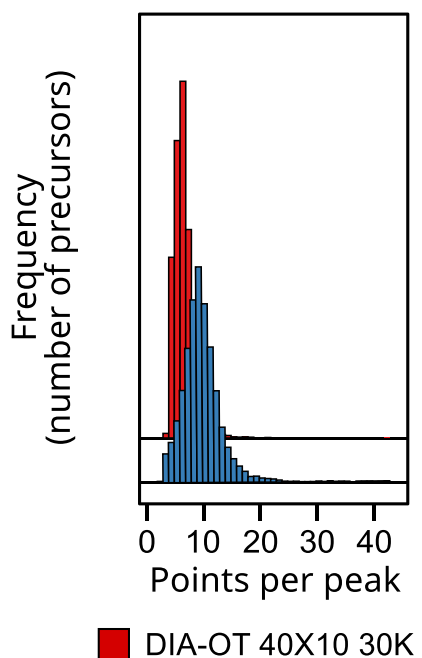

$1 \mu \mathrm{g}$ input

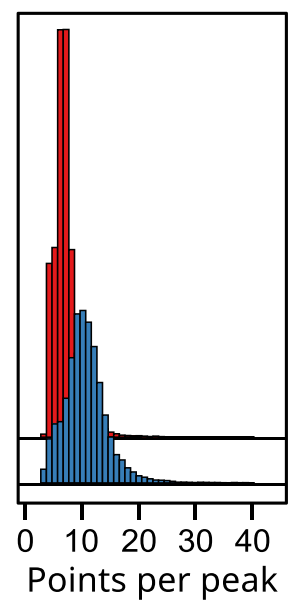

DIA-IT 40X10 normal
$G$

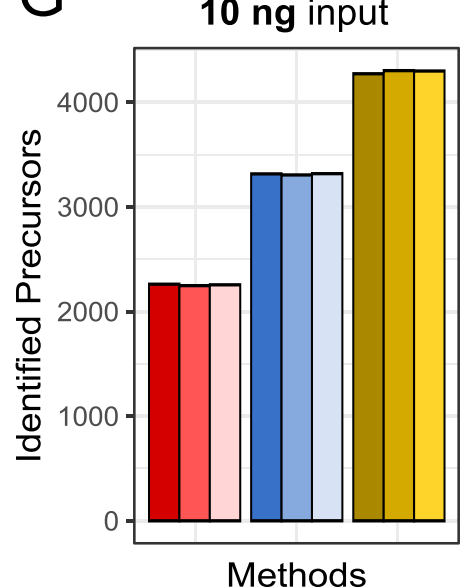

DIA-OT 40X10 30K DIA-IT 40X10 normal

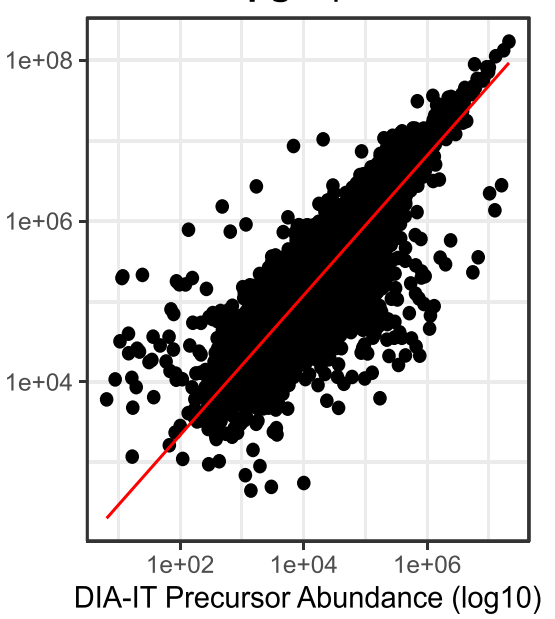

Figure 1. (A) Comparison of number of peptide ions identified among the reference DIA-OT method (DIA-IT 40X10 30K) and three DIA-IT $40 \mathrm{X} 10$ methods at different scanning speeds (normal, rapid, turbo) when analyzing $1 \mathrm{ng}, 10 \mathrm{ng}, 100 \mathrm{ng}$, and $1 \mu \mathrm{g}$ of HeLa protein digest. (B) Comparison of the number of peptide ions identified when performing triplicate analyses of $1 \mathrm{ng}$ of HeLa protein digest with the reference DIA-OT method (DIA-OT 40X10 30K) and three DIA-IT 40X10 methods at different scanning speeds (normal, rapid, turbo). (C) Overlap of the set of peptide sequences identified in triplicate analyses between the reference DIA-OT method (DIA-IT 40X10 30K) and DIA-IT 40X10 normal scan rate when analyzing $10 \mathrm{ng}$ and $1 \mu \mathrm{g}$ of HeLa protein digest. (D) Distribution of coefficient of variation of the DIA-OT 40X10 30K and the DIA-IT $40 \mathrm{X} 10$ normal methods when analyzing $10 \mathrm{ng}$ and $1 \mu \mathrm{g}$ of HeLa protein digest in triplicate. (E) Correlation of peptide abundances obtained from 
Figure 1. continued

the average area of the six most intense fragment ions in the analysis of $10 \mathrm{ng}$ and $1 \mu \mathrm{g}$ HeLa protein digest using the DIA-OT method (DIA-OT $40 \mathrm{X} 1030 \mathrm{~K}$ ) and DIA-IT 40X10 normal scan rate. (F) Distribution of the number of sampling points per chromatographic peak in the reference DIA-OT method (DIA-OT 40X10 30K) and DIA-IT 40X10 normal scan rate. (G) Number of peptide ions identified when performing triplicate analyses of $10 \mathrm{ng}$ and $1 \mu \mathrm{g}$ of HeLa protein digest with the DIA-OT method (DIA-OT 40X10 30K) and the DIA-IT methods using a large (10 Da) and small (4 Da) isolation windows (DIA-IT 40X10 and DIA-IT 100X4, respectively).

we set a DIA-IT method with a large number of small isolation windows, i.e., 100 windows of $4 \mathrm{Da}$ covering the range of 500$900 \mathrm{~m} / z$, to improve the method specificity, minimize fragment ion interferences, and facilitate peptide identification. We used this method to analyze the former serial dilutions of HeLa protein digest $(1,10,100$, and $1000 \mathrm{ng})$ and compared it to the previously evaluated DIA-IT and DIA-OT methods bearing an isolation scheme of 40 windows of $10 \mathrm{Da}$ each. Our results showed a consistent increase in the number of identified peptides in all DIA-IT methods when using a large number of small isolation windows, independently of the scanning mode (normal, rapid, turbo) and the protein amount being analyzed (Figure 1G, Supplementary Figure 4D). Moreover, the higher specificity of DIA-IT 100X4 methods enable them to maintain a similar coefficient of variation in triplicate analyses than DIAIT 40X10 methods, despite the increase in cycle time (Supplementary Figure 4E).

Overall our results demonstrate the benefits of using linear ion trap-based DIA methods-especially their high-specificity variant (100 windows of $4 \mathrm{Da}$ each) - to improve the number of identified peptides in low-input proteomes. The high sampling rates exhibited by some DIA-IT methods (e.g., from $1.1 \mathrm{~s}$ in turbo to $1.9 \mathrm{~s}$ in normal) makes them not only compatible with low-input applications but also with ion mobility separation to further increase their specificity and with the ultrafast chromatography separations recently adopted in the field for high-throughput translational proteomics. ${ }^{22,25-27}$ In conclusion, the use of linear ion trap-based DIA methods benefits peptide identifications in low-input proteomics applications, and together with recent analytical advances, they might further contribute to the feasibility of single cell proteomics.

The mass spectrometry proteomics data obtained from DIAIT and all its companion methods have been deposited at the ProteomeXChange Consortium via the PRIDE repository with identifier PXD025766. ${ }^{28}$

\section{ASSOCIATED CONTENT}

\section{SI Supporting Information}

The Supporting Information is available free of charge at https://pubs.acs.org/doi/10.1021/acs.analchem.1c01885.

Detailed materials and methods. Supplementary Figure 1: Precursor abundances of identified peptides with different DIA methods and correlation of their retention time values. Supplementary Figure 2: Comparison of the predicted spectra and experimental pseudo-MS2 spectra. Supplementary Figure 3: Number of annotated fragments per precursor in the experimental pseudo-MS2 spectra. Supplementary Figure 4: Number of sampling points per chromatographic peak in the different DIA methods. (PDF)

Supplementary Table 1: List of precursors identified by directDIA in DIA-OT $40 \mathrm{X} 1030 \mathrm{~K}$ in $1 \mu \mathrm{g}$ HeLa protein digest (XLSX)
Supplementary Table 2: List of precursors identified by directDIA in DIA-OT $40 \mathrm{X} 1030 \mathrm{~K}$ in $10 \mathrm{ng} \mathrm{HeLa}$ protein digest (XLSX)

Supplementary Table 3: List of precursors identified by directDIA in DIA-IT 40X10 normal in $1 \mu \mathrm{g}$ HeLa protein digest (XLSX)

Supplementary Table 4: List of precursors identified by directDIA in DIA-IT 40X10 normal in $10 \mathrm{ng} \mathrm{HeLa}$ protein digest (XLSX)

Supplementary Table 5: List of precursors identified by directDIA in DIA-IT 100X4 normal in $1 \mu \mathrm{g}$ HeLa protein digest (XLSX)

Supplementary Table 6: List of precursors identified by directDIA in DIA-IT 100X4 normal in $10 \mathrm{ng}$ HeLa protein digest (XLSX)

\section{AUTHOR INFORMATION}

\section{Corresponding Author}

Eduard Sabidó - Center for Genomic Regulation, Barcelona Institute of Science and Technology (BIST), 08003

Barcelona, Spain; Universitat Pompeu Fabra, 08003

Barcelona, Spain; 이이이.org/0000-0001-6506-7714; Email: eduard.sabido@crg.cat

\section{Authors}

Eva Borràs - Center for Genomic Regulation, Barcelona Institute of Science and Technology (BIST), 08003 Barcelona, Spain; Universitat Pompeu Fabra, 08003 Barcelona, Spain

Olga Pastor - Center for Genomic Regulation, Barcelona Institute of Science and Technology (BIST), 08003 Barcelona, Spain; Universitat Pompeu Fabra, 08003 Barcelona, Spain

Complete contact information is available at: https://pubs.acs.org/10.1021/acs.analchem.1c01885

\section{Notes}

The authors declare no competing financial interest.

\section{ACKNOWLEDGMENTS}

The CRG/UPF Proteomics Unit is part of the Spanish Infrastructure for Omics Technologies (ICTS OmicsTech) and it is member of ProteoRed PRB3 consortium which is supported by Grant PT17/0019 of the PE I+D+i 2013-2016 from the Instituto de Salud Carlos III (ISCIII), ERDF, and "Secretaria d'Universitats i Recerca del Departament d'Economia i Coneixement de la Generalitat de Catalunya" (2017SGR595). This project was supported by EPIC-XS, Project Number 823839, funded by the Horizon 2020 programme of the European Union. We also acknowledge support of the Spanish Ministry of Science and Innovation to the EMBL partnership, the Centro de Excelencia Severo Ochoa, and the CERCA Programme/Generalitat de Catalunya. 


\section{REFERENCES}

(1) Regev, A.; Teichmann, S. A.; Lander, E. S.; Amit, I.; Benoist, C.; Birney, E.; Bodenmiller, B.; Campbell, P.; Carninci, P.; Clatworthy, M.; Clevers, H.; Deplancke, B.; Dunham, I.; Eberwine, J.; Eils, R.; Enard, W.; Farmer, A.; Fugger, L.; Göttgens, B.; Hacohen, N.; Haniffa, M.; Hemberg, M.; Kim, S.; Klenerman, P.; Kriegstein, A.; Lein, E.; Linnarsson, S.; Lundberg, E.; Lundeberg, J.; Majumder, P.; Marioni, J. C.; Merad, M.; Mhlanga, M.; Nawijn, M.; Netea, M.; Nolan, G.; Pe'er, D.; Phillipakis, A.; Ponting, C. P.; Quake, S.; Reik, W.; Rozenblatt-Rosen, O.; Sanes, J.; Satija, R.; Schumacher, T. N.; Shalek, A.; Shapiro, E.; Sharma, P.; Shin, J. W.; Stegle, O.; Stratton, M.; Stubbington, M. J. T.; Theis, F. J.; Uhlen, M.; van Oudenaarden, A.; Wagner, A.; Watt, F.; Weissman, J.; Wold, B.; Xavier, R.; Yosef, N. eLife 2017, 6, na DOI: 10.7554/eLife.27041.

(2) Stuart, T.; Satija, R. Nat. Rev. Genet. 2019, 20 (5), 257-272.

(3) Brunner, A.-D.; Thielert, M.; Vasilopoulou, C. G.; Ammar, C.; Coscia, F.; Mund, A.; Hoerning, O. B.; Bache, N.; Apalategui, A.; Lubeck, M.; Richter, S.; Fischer, D. S.; Raether, O.; Park, M. A.; Meier, F.; Theis, F. J.; Mann, M. Ultra-High Sensitivity Mass Spectrometry Quantifies Single-Cell Proteome Changes upon Perturbation. bioRxiv preprint, 2020. DOI: 10.1101/ 2020.12.22.423933.

(4) Stejskal, K.; Op de Beeck, J.; Dürnberger, G.; Jacobs, P.; Mechtler, K. Anal. Chem. 2021, 93 (25), 8704-8710.

(5) Zhu, Y.; Piehowski, P. D.; Zhao, R.; Chen, J.; Shen, Y.; Moore, R. J.; Shukla, A. K.; Petyuk, V. A.; Campbell-Thompson, M.; Mathews, C. E.; Smith, R. D.; Qian, W.-J.; Kelly, R. T. Nat. Commun. 2018, 9 (1), 882.

(6) Demichev, V.; Yu, F.; Teo, G. C.; Szyrwiel, L.; Rosenberger, G. A.; Decker, J.; Kaspar-Schoenefeld, S.; Lilley, K. S.; Mülleder, M.; Nesvizhskii, A. I.; Ralser, M. High Sensitivity Dia-PASEF Proteomics with DIA-NN and FragPipe. bioRxiv preprint, 2021. DOI: 10.1101/ 2021.03.08.434385.

(7) Budnik, B.; Levy, E.; Harmange, G.; Slavov, N. Genome Biol. 2018, 19 (1), 161.

(8) Marx, V. Nat. Methods 2019, 16 (9), 809-812.

(9) Borràs, E.; Sabidó, E. Proteomics 2017, 17 (17-18), 1700180.

(10) Ludwig, C.; Gillet, L.; Rosenberger, G.; Amon, S.; Collins, B. C.; Aebersold, R. Mol. Syst. Biol. 2018, 14 (8), e8126.

(11) Gotti, C.; Roux-Dalvai, F.; Joly-Beauparlant, C.; Leclercq, M.; Mangnier, L.; Droit, A. Extensive and Accurate Benchmarking of DIA Acquisition Methods and Software Tools Using a Complex Proteomic Standard. bioRxiv preprint, 2020. DOI: 10.1101/2020.11.03.365585.

(12) Venable, J. D.; Dong, M.-Q.; Wohlschlegel, J.; Dillin, A.; Yates, J. R. Nat. Methods 2004, 1 (1), 39-45.

(13) Panchaud, A.; Scherl, A.; Shaffer, S. A.; von Haller, P. D.; Kulasekara, H. D.; Miller, S. I.; Goodlett, D. R. Anal. Chem. 2009, 81 (15), 6481-6488.

(14) Gillet, L. C.; Navarro, P.; Tate, S.; Röst, H.; Selevsek, N.; Reiter, L.; Bonner, R.; Aebersold, R. Mol. Cell Proteomics 2012, 11 (6), O111.016717.

(15) Moseley, M. A.; Hughes, C. J.; Juvvadi, P. R.; Soderblom, E. J.; Lennon, S.; Perkins, S. R.; Thompson, J. W.; Steinbach, W. J.; Geromanos, S. J.; Wildgoose, J.; Langridge, J. I.; Richardson, K.; Vissers, J. P. C. J. Proteome Res. 2018, 17 (2), 770-779.

(16) Bruderer, R.; Bernhardt, O. M.; Gandhi, T.; Xuan, Y.; Sondermann, J.; Schmidt, M.; Gomez-Varela, D.; Reiter, L. Mol. Cell Proteomics 2017, 16 (12), 2296-2309.

(17) Plumb, R. S.; Johnson, K. A.; Rainville, P.; Smith, B. W.; Wilson, I. D.; Castro-Perez, J. M.; Nicholson, J. K. Rapid Commun. Mass Spectrom. 2006, 20 (13), 1989-1994.

(18) Meier, F.; Brunner, A.-D.; Frank, M.; Ha, A.; Bludau, I.; Voytik, E.; Kaspar-Schoenefeld, S.; Lubeck, M.; Raether, O.; Bache, N.; Aebersold, R.; Collins, B. C.; Röst, H. L.; Mann, M. Nat. Methods 2020, 17 (12), 1229-1236.

(19) Kiyonami, R.; Senko, M.; Zabrouskov, V.; Hühmer, A.; Egertson, J.; Ting, S.; MacCoss, M. Large-Scale Targeted Protein Quantification Using WiSIM-DIA on an Orbitrap Fusion Tribrid Mass
Spectrometer; Thermo Application Note 600; No. AN64026 E 09/ 16S; ThermoFisher, 2014.

(20) Espadas, G.; Borràs, E.; Chiva, C.; Sabidó, E. Proteomics 2017, 17 (9), 1600416.

(21) Trujillo, E. A.; Hebert, A. S.; Brademan, D. R.; Coon, J. J. Anal. Chem. 2019, 91 (20), 12625-12629.

(22) Bekker-Jensen, D. B.; Bernhardt, O. M.; Hogrebe, A.; MartinezVal, A.; Verbeke, L.; Gandhi, T.; Kelstrup, C. D.; Reiter, L.; Olsen, J. V. Nat. Commun. 2020, 11 (1), 787.

(23) Borràs, E.; Sabidó, E. Anal. Chem. 2018, 90 (21), 1233912341.

(24) Heil, L. R.; Remes, P. M.; MacCoss, M. J. Comparison of Unit Resolution versus High-Resolution Accurate Mass for Parallel Reaction Monitoring. bioRxiv preprint, 2021. DOI: 10.1101/ 2021.05.04.442680.

(25) Bache, N.; Geyer, P. E.; Bekker-Jensen, D. B.; Hoerning, O.; Falkenby, L.; Treit, P. V.; Doll, S.; Paron, I.; Müller, J. B.; Meier, F.; Olsen, J. V.; Vorm, O.; Mann, M. Mol. Cell Proteomics 2018, 17 (11), 2284-2296.

(26) Bian, Y.; Zheng, R.; Bayer, F. P.; Wong, C.; Chang, Y.-C.; Meng, C.; Zolg, D. P.; Reinecke, M.; Zecha, J.; Wiechmann, S.; Heinzlmeir, S.; Scherr, J.; Hemmer, B.; Baynham, M.; Gingras, A.-C.; Boychenko, O.; Kuster, B. Nat. Commun. 2020, 11 (1), 157.

(27) Bekker-Jensen, D. B.; Martínez-Val, A.; Steigerwald, S.; Rüther, P.; Fort, K. L.; Arrey, T. N.; Harder, A.; Makarov, A.; Olsen, J. V. Molecular \& Cellular Proteomics 2020, 19 (4), 716-729.

(28) Vizcaíno, J. A.; Deutsch, E. W.; Wang, R.; Csordas, A.; Reisinger, F.; Ríos, D.; Dianes, J. A.; Sun, Z.; Farrah, T.; Bandeira, N.; Binz, P.-A.; Xenarios, I.; Eisenacher, M.; Mayer, G.; Gatto, L.; Campos, A.; Chalkley, R. J.; Kraus, H.-J.; Albar, J. P.; MartinezBartolomé, S.; Apweiler, R.; Omenn, G. S.; Martens, L.; Jones, A. R.; Hermjakob, H. Nat. Biotechnol. 2014, 32 (3), 223-226. 